\title{
ESTRATEGIAS PARA EL DESARROLLO DE LA COMPETENCIA COMUNICATIVA EN LA FASE DE LA PREESCRITURA
}

\author{
Marta Madrigal Abarca
}

\begin{abstract}
RESUMEN
El proceso de llevar a la forma escrita conceptos, ideas y pensamientos a veces es muy complejo, ya que un texto escrito representa el producto de una serie de operaciones mentales complicadas. Empezar es, a menudo, la parte más difícil, aún si el escritor tiene en mente lo que quiere decir. Sin embargo, existen diferentes recursos útiles para facilitar este proceso. El propósito de este artículo es mostrar algunas técnicas realizadas en la etapa de la preescritura para la planificación de un texto escrito. Estas estrategias desarrolladas mediante diferentes formas -escritura rápida, gráficos o diagramas- pueden ayudar al escritor a lograr un producto textual cohesionado y coherente.

Palabras clave: preescritura, planificación, lluvia de ideas, proceso de escritura.
\end{abstract}

\begin{abstract}
The process to the written form concepts, ideas and thoughts at times is very complex, as a written text is the product of a series of complex mental operations. Start is often the hardest part, even if the author has in mind what you mean. However, there are various useful resources to facilitate this process. The purpose of this article is to show some techniques under the phase of the pre-writing for the planning of a written text. These strategies developed through different forms - quick writing, graphics or diagrams- can help the writer to achieve a product textual cohesive and coherent.
\end{abstract}

Key words: pre-writing, planning, plethora of ideas, writing process.

\section{Introducción}

La elaboración de un texto escrito coherente lleva un tiempo de trabajo y práctica, ya que requiere la atención no solo de los aspectos formales de gramática, puntuación y acentuación sino también del contenido y del contexto en que se da el hecho comunicativo. Estos razonamientos son la causa por la cual la escritura es considerada como una práctica difícil, compleja y hasta agotadora.

M.L. Marta Madrigal Abarca. Profesora de la Escuela de Filología, Lingüística y Literatura. Universidad de Costa Rica.

Correo electrónico: madrigal_ma@yahoo.com.mx

Recepción: 03- 06- 2011

Aceptación: 23- 08- 2011 
Muchas veces, una de las partes más arduas del proceso es cómo empezar a escribir. Puede ser que las ideas lleguen, pero no se sabe cómo organizarlas, cómo encontrar las ideas principales, cómo distinguir las secundarias y cómo unirlas para tener un texto cohesionado y coherente.

Sin embargo, el trabajo se vuelve más fácil cuando se planifican los textos, cuando se toma un tiempo previo a la escritura para reflexionar, organizar y distribuir las piezas textuales. Muchos estudiantes evitan este paso, porque lo consideran innecesario; no han aprendido que, antes de hacer un borrador, la tarea les va a resultar más fácil y divertida si ha sido previamente planificada y, de acuerdo con Arley Fonseca (2010), esta perspectiva lúdica también es importante para desarrollar la capacidad creativa e innovadora de los escritores en la práctica de la escritura.

\section{Justificación y objetivos}

El objetivo de este artículo es mostrar algunas técnicas útiles para planificar el texto antes de empezar el proceso de textualización. Son estrategias que pueden cumplir con varios propósitos: desde encontrar ideas hasta organizarlas, desde la elaboración de una oración temática o de cualquier tipo de párrafo hasta el desarrollo de una secuencia textual sea descriptiva, narrativa, expositiva o argumentativa.

Cada una de las técnicas está ejemplificada con muestras hechas por estudiantes, provenientes de diversas áreas de estudio, en el Curso básico de redacción de la Universidad de Costa Rica.

Los modelos desarrollan la búsqueda de ideas y su posible organización en la creación del borrador, de modo que la escritura de los diferentes párrafos es preliminar, es decir, no están corregidos ni pulidos como un producto final. El único propósito es, entonces, desarrollar la fase de la preescritura mediante ejercicios creativos como la elaboración de gráficos, figuras y diagramas, entre otros.

\section{Marco teórico}

\subsection{El discurso y la comunicación}

El discurso, ya sea oral o escrito, es una práctica social que se articula a partir del uso lingüístico contextualizado; es decir, cuando se habla o se escribe, lo que se hace es construir fragmentos textuales orientados a alcanzar ciertos fines que se dan en interdependencia con el contexto lingüístico, cognitivo y sociocultural.

Desde el punto de vista lingüístico, la lengua es la materia prima del discurso; los elementos que la conforman constituyen un conjunto de estructuras y funciones que establecen entre ellas diversas combinaciones y relaciones con el propósito de producir mensajes; esto es, establecer una comunicación. Así, la lengua ofrece a quienes la usan una serie de opciones fónicas, gráficas, morfosintácticas y léxicas.

El hablante, en el momento de interactuar discursivamente, debe escoger consciente o inconscientemente entre esas opciones. Sin embargo, esta elección no se realiza al azar, sino de acuerdo con unos parámetros contextuales que incluyen la situación, los propósitos de quien la realiza y las características de los destinatarios, entre otros. 
Entonces, como práctica social lo que se persigue con el discurso es la comunicación, por lo que este, aunque sea complejo y heterogéneo, no es caótico.

\footnotetext{
La heterogeneidad lingüístico discursiva no es lo mismo que caótica sino que está regulada más allá del plano gramatical por una serie de normas, principios o máximas de carácter textual y sociocultural que orientan a las personas en la tarea de construir piezas discursivas coherentes y apropiadas a cada ocasión de comunicación, la cual es entendida no solo como un simple y mecánico proceso de transmisión de información entre dos polos, sino como un proceso interactivo mucho más complejo que incluye la continua interpretación de intenciones expresadas verbal y no verbalmente, de forma directa o velada. (Calsamiglia y Tusón 1999: 16)
}

Las personas, como miembros de una sociedad, tienen una ideología, una visión de mundo y como tal tienen unas intenciones, metas o finalidades concretas en cada situación y ponen en práctica una diversidad de estrategias para la consecución de esos fines. De la misma manera, en la práctica de la escritura, el texto debe desarrollarse con miras a cumplir las expectativas que el autor se ha planteado, pues ha sido creado con el objetivo de comunicar.

Al respecto, Valdés, Dvorak y Pagán (1998) señalan que escribir es un intento de comunicar una idea a otra u otras personas por una razón específica. La meta del escritor es enviar un mensaje para convencer, criticar, explicar, entretener, informar, preguntar o recomendar, entre otros. El lector, por su parte, también tiene un propósito: obtener la información para tomar una decisión, para saber que comparte con otros su propias ideas, para entretenerse, etc. Por ello, se puede afirmar que el éxito de la comunicación escrita depende del establecimiento de un propósito compartido entre escritor y lector. Esto es, el escritor anticipa las respuestas a las posibles preguntas que el lector se formula con relación al texto.

\subsection{La adquisición de la competencia escrita}

A menudo, afirma Cassany (1989: 27), el código escrito es considerado como un sistema de signos por el cual se transcribe el código oral, sin tomar en cuenta que cada uno tiene sus propias características. Pero, gracias a los estudios realizados sobre la adquisición del lenguaje, se sabe que la adquisición de ambos códigos no sigue el mismo proceso. Adquirir el código escrito no es solo aprender la correspondencia entre el sonido y la grafía, sino también aprender un nuevo código diferente al oral; es un aprendizaje institucionalizado y esta condición es una de sus principales características. Asimismo, desde la perspectiva de Calsamiglia y Tusón:

\footnotetext{
La característica más importante de la adquisición de la competencia escrita es que está sometida a un aprendizaje institucionalizado, que tiene lugar en centros de instrucción y educación. A pesar de que la lengua escrita está presente en el entorno cotidiano, el aprendizaje del código exige un adiestramiento y una preparación específica. (1999: 78)
}

De esta manera, en el mundo moderno, la capacidad de leer (en el sentido de comprender, contextualizar, interpretar) textos elaborados y la capacidad de escribir para dar cuenta de la adquisición de estos conocimientos se ha convertido en el eje primordial de la instrucción.

\subsection{El proceso de escritura}

A través de los años, la enseñanza de la escritura ha sido abordada desde una gran diversidad de métodos como el enfoque basado en el producto o el enfoque basado en la 
gramática, los cuales generaron variadas estrategias metodológicas; no obstante, se orientan a explicaciones teóricas y ejercicios de ortografía, morfología y sintaxis.

Estas metodologías no son inapropiadas, pues las reglas ortográficas, morfosintácticas y léxicas permiten construir oraciones correctas, pero estos componentes son solo una parte del conjunto de conocimientos que domina el usuario y no necesariamente permiten la elaboración de textos cohesionados y coherentes, ya que estos criterios tienen sus propias reglas. De manera que, para que la comunicación escrita sea exitosa, se requiere tanto de los aspectos formales como de contenido, es decir, no solo del uso de la ortografía, la morfosintaxis y el léxico apropiados como también de la adecuación, la coherencia y la cohesión.

Ahora bien, en los últimos años se han planteado nuevas formas de favorecer el aprendizaje de esta competencia, gracias a los avances en psicología cognitiva, pragmática y lingüística textual. Entre las teorías más destacadas se encuentran las formuladas por Van Dijk -relacionadas más estrechamente con los estudios de lingüística textual-, así como las concepciones de G. Rohman y las de Hayes y Flower que están más próximas a la psicología cognitiva.

De acuerdo con esas teorías, cuando el hablante se expresa, lee, escucha o escribe construye textos, sin embargo, para poder hacerlo de manera eficiente debe dominar habilidades más allá de la ejecución de simples ejercicios mecánicos. Al escribirse un mensaje, sin importar su naturaleza ni su forma, es necesario cumplir con una serie de condiciones y requisitos antes de fijarlo en el papel; quien escribe debe tener claro el contenido del conjunto del mensaje y su articulación, tanto en lo conceptual como en lo expositivo.

Por esta razón, es indispensable que, previo a la escritura, se planifique el texto de manera que se diferencie la idea central de las secundarias, se reconozcan las premisas y las conclusiones, se identifiquen las relaciones lógicas entre las proposiciones, se elija el criterio para organizar la presentación de las ideas, se determinen los pasos para el desarrollo de la idea central y los conceptos generales y se establezca el modo de presentación de las ideas secundarias y los ejemplos específicos, entre otros.

Como lo anota Bolaños (2002), antes de empezar a escribir el texto, es necesario disponer de algún esquema de organización, un conjunto de elementos parciales que se irán concatenando y desarrollando detalladamente y con precisión conforme va tomando cuerpo la estructura textual que se quiere producir. En otras palabras, es un proceso en el cual se va uniendo uno por uno los elementos que determinan lo que escribimos (Brooke y Grundy 1998).

\subsection{El modelo del proceso}

Durante los últimos años, ha habido una tendencia de investigar qué hacen los escritores cuando desarrollan una actividad discursiva, lo cual ha generado una gran cantidad de modelos o métodos que intentan explicar el funcionamiento del proceso de composición; entre los más relevantes están los cognitivos, los cuales describen las diferentes operaciones intelectuales que realiza un autor para escribir un texto.

Desde esta perspectiva, uno de los modelos más completos es el desarrollado por Linda Flower y John R. Hayes (1980 y 1981), el cual está formado por distintos procesos y subprocesos mentales básicos, con una organización jerárquica y con unas determinadas reglas de funcionamiento. El modelo de Flower y Hayes consta de tres grandes unidades: la situación de comunicación, la memoria a largo plazo del escritor y los procesos de escritura. 
La primera unidad -la situación de la comunicación- incluye todos los elementos externos del escritor como el problema retórico (tema, audiencia, propósito canal, etc.), mientras que la segunda y la tercera unidad ocurren en lo interno del autor, en su cerebro. Así, la memoria a largo plazo se refiere al almacenamiento de todos los conocimientos sobre el tema del texto, la audiencia o los diversos tipos de textos que se pueden escribir y los procesos de escritura se relacionan con las fases de planificar, redactar y examinar, las cuales corresponden a las tres etapas del llamado Modelo del proceso que son preescritura, borrador y revisión.

Según los seguidores de este modelo, el buen escritor es aquella persona que pasa por el proceso de reflexión, textualización y corrección durante el desarrollo de su escrito. Cada una de las partes es importante, porque cada una cumple una función específica.

En la preescritura, se buscan los temas, se generan ideas, se va organizando el texto, mientras que en la fase del borrador o proceso de textualización se escriben, de manera preliminar, las ideas obtenidas en la etapa de la preescritura. En esta fase, se ordenan las ideas que se han generado en la planificación, mediante la formación de párrafos, la selección de la información pertinente, la estructura lógica de los datos, el empleo de las oraciones temáticas y la puntuación correcta. Por último, en la etapa de la revisión se evalúa lo escrito tanto en lo que respecta a la forma como en lo concerniente al contenido (Madrigal Abarca 2005).

\subsection{Tipología de escritores}

Los investigadores que enfatizan el proceso de composición establecen la diferencia entre los escritores competentes y los no competentes. Los primeros han desarrollado una variada gama de estrategias que les permite expresar inteligiblemente sus ideas: realizan esquemas, escriben borradores, releen lo escrito, etc. En cambio, los segundos no han llegado a dominar estas microhabilidades piensan que la escritura de un texto consiste simplemente en anotar en un papel las ideas a medida que se les va ocurriendo. Y, como lo anota Cassany: "[...] creen que un texto escrito es espontáneo como uno oral, que no hace falta reelaborarlo ni revisarlo. En definitiva, no han aprendido a generar ideas, a enriquecerlas, a organizarlas para un lector y a traducirlas al código escrito" (1989: 102).

A pesar de que el pasar de una tipología a otra es difícil, no es imposible. Se logra con práctica, con trabajo y con mucha dedicación. La habilidad de escribir constituye todo un aprendizaje, no es espontánea ni innata; tampoco es un acto de intuición (Merino 2007). De acuerdo con Carolina Figueras y Marisa Santiago (2008), cualquier hablante puede expresarse en su lengua de manera comprensible aunque nunca haya asistido a la escuela, pero ningún hablante tiene la capacidad de escribir sin haber tenido algún periodo de estudio y práctica de esta destreza.

Está claro, entonces, que elaborar textos correctos, adecuados y que cumplan con el objetivo para el cual fueron creados no es fruto de la casualidad, sino de la reflexión y del trabajo; ello significa que las estrategias para ser escritores competentes pueden ser aprendidas y mejoradas en la práctica. Asimismo, redactar un texto tomando en cuenta el proceso supone el desarrollo de cada una de sus etapas. Estas no son unitarias ni rígidas, ni se suceden de manera lineal siguiendo un orden determinado, sino que es el mismo autor quien las ordena y organiza según sus objetivos, de manera tal que cada parte del proceso puede realizarse más de una vez, en cualquier momento de la composición.

Cada una de estas etapas es imprescindible, sin embargo, en este artículo solo se muestran algunas actividades que pueden realizarse en la preescritura, mediante diferentes técnicas. 


\section{La preescritura: la planificación del texto}

La planificación es el proceso realizado previo a la escritura; frecuentemente es omitido por la mayoría de escritores inexpertos, ya que les parece innecesario. Este tipo de escritor empieza a redactar sobre un tema sin haber meditado sobre lo que va a decir; expone las ideas tal como le van llegando a su mente, en consecuencia, resulta un texto desorganizado, un escrito que no es adecuado a las expectativas del lector, un texto que no funciona para lo que fue creado. Por ello, la preescritura se convierte en una práctica fundamental antes de empezar a escribir.

Se trata de encontrar ideas sobre el tema, pensar en esas ideas y cómo pueden interactuar entre sí, cómo se desarrollan y se organizan (Madrigal Abarca 2008). Además, se debe reflexionar en las circunstancias en que se produce el texto, en las características de la situación comunicativa en la que se inscribe. Carolina Figueras y Marisa Santiago (2000: 15) denominan este conjunto de circunstancias como "problema retórico", es decir, que para producir textos apropiados para cada situación comunicativa es conveniente plantearse las siguientes preguntas:

a. Objetivos del texto: para qué se escribe el texto, qué objetivo hay que lograr con el texto.

b. El texto como relación: a quién se dirige el texto, qué sabe exactamente el lector, cómo quiere presentarse el escritor ante el destinatario, qué imagen pretende transmitirle.

c. Organización de las ideas: qué debe decir el texto exactamente, cómo se organizará la información para que la comunicación sea más eficaz.

d. Géneros: en qué género se inscribe el texto, cuáles son las convenciones asociadas al género escogido.

e. Secuencias textuales básicas: qué tipo de secuencias textuales vamos a emplear: descriptivas, narrativas, expositivas, argumentativas.

\subsection{Diferentes recursos de planificación textual}

Los hábitos de escritura, así como sus métodos, son tan individuales como los escritores. Es poco frecuente que un escritor pueda ir desde la introducción hasta la conclusión de su escrito de una manera espontánea y casi automática; la mayoría de ellos escriben muchos borradores antes de tener uno que les satisfaga. Escribir es una actividad compleja, por eso es recomendable dividir el trabajo en estadios y así concentrarse en una tarea en un tiempo y no en todo a la vez.

Por otra parte, en el acto de la escritura, empezar es la parte más difícil aún cuando se sepa que es lo que se quiere decir. A menudo se necesita un calentamiento previo, especialmente cuando quien escribe no cuenta todavía con mucha práctica. Para facilitar este proceso existen varios recursos, las autoras Lisa Gerrard y Sheri Spaine (1993) describen dos de ellos: la escritura automática o escritura libre (en inglés, freewriting) y la lluvia de ideas (en inglés, brainstorming).

El primero, la escritura automática, consiste en escribir continuamente, por un periodo específico de tiempo, lo que venga a la mente, sin detenerse a corregir, a leer o pensar en lo que se está escribiendo. Según las autoras mencionadas, este recurso es muy útil, ya que entrena a los escritores a desarrollar sus ideas espontáneamente. Como estos no tienen que preocuparse por lo que van a decir ni cómo lo van decir, las ideas fluyen fácilmente. La 
puesta en práctica diaria de este ejercicio es un buen recurso para desarrollar la fluidez en los escritores todavía inexpertos.

La lluvia de ideas consiste en unir cada idea en un mismo tema. Es decir, se trata de crear listados de palabras o frases cortas relacionadas con el tema en común. De esta manera, se puede obtener un amplio rango de ideas posibles, las cuales serán usadas en el proceso de textualización. Tanto la escritura automática como la lluvia de ideas son útiles como simples forma de "calentamiento" o como recursos para obtener y organizar ideas.

Esas técnicas pueden desarrollarse mediante diversas formas, gráficos o diagramas como estrellas, óvalos, cubos o árboles. Algunas de ellas son: el diagrama de Venn, la estrella de palabras, la rueda de palabras, el árbol de ideas y el cubo.

Los siguientes apartados muestran estos recursos empleados por los estudiantes de un grupo del Curso básico de redacción de la Universidad de Costa Rica.

\subsubsection{Diagrama de Venn}

Los diagramas de Venn son ilustraciones usadas en Matemáticas y en Lógica. La técnica consiste en agrupar elementos en conjuntos mediante dos círculos u óvalos entrecruzados o solapados. El área central contiene los elementos comunes de ambos círculos. Actualmente, este tipo de diagrama es empleado en la etapa de la preescritura cuando se quiere enfatizar el proceso; resulta muy útil, ya que agrupa las ideas en forma de contraste, razón por la cual es muy usado para escribir párrafos de comparación y para construir textos argumentativos (Gerrard y Spaine 1993).

El siguiente modelo, elaborado a partir del trabajo realizado por un estudiante, muestra una búsqueda de ideas para la escritura de un párrafo de comparación entre las universidades públicas y las universidades privadas en Costa Rica.

$$
\text { Universidad pública }
$$

Universidad privada

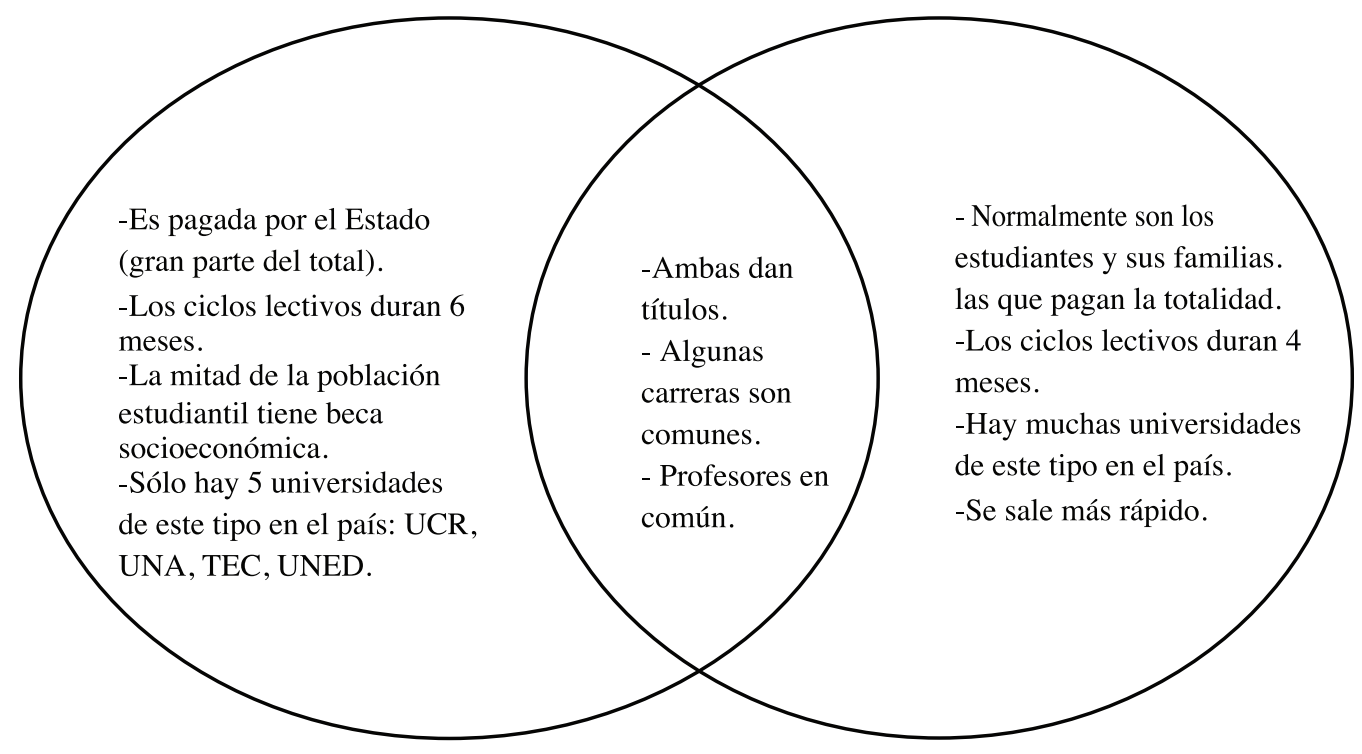

Figura 1. Ejemplo del diagrama de Venn. Autora, con base en el trabajo de un estudiante 
El párrafo resultante es el siguiente:

La educación superior en Costa Rica consta de universidades públicas como UCR, UNA, UNED y el TEC y de universidades privadas como Ulacit, ULatina, UMCA, Hispanoamericana, Veritas, entre otras. En el primer tipo de universidades, los ciclos lectivos duran seis meses; en cambio en las universidades privadas duran cuatro meses, lo cual provoca que en estas se obtenga un título más rápido. Por otra parte, las universidades públicas son más baratas que las privadas, pues en las públicas se financian con fondos públicos, a diferencia de las privadas en donde se debe pagar grandes sumas de dinero para poder ingresar a ellas. Sin embargo, estos dos tipos de educación superior tienen puntos en común, por ejemplo, las dos, al final de la carrera, le entregan al estudiante un título académico que le permitirá trabajar como profesional en cualquier parte del país. Además, muchos profesores que trabajan en las universidades públicas, también desempeñan labores docentes en las universidades privadas. Como último punto común que se puede mencionar es que muchas de las carreras que se dan en ambos tipos de universidades son las mismas, por ejemplo, Arquitectura, Ingenierías, Administración de negocios, Medicina, Odontología, entre otras.

\subsubsection{La estrella de palabras}

Un diagrama de estrellas ayuda a la generación de ideas mediante listados de palabras y de oraciones. El procedimiento es el siguiente: se dibuja una estrella de cinco puntas y en el centro de ella se escribe el tema que se quiere desarrollar después en el borrador. Luego, en cada punta, se escribe una palabra -o una oración- que puede ser asociada con ese tema (Gerrard y Spaine 1993).

En la clase, este ejercicio fue realizado de la siguiente manera: el estudiante recibió una tarjeta con una imagen. El propósito era hacer un párrafo descriptivo.

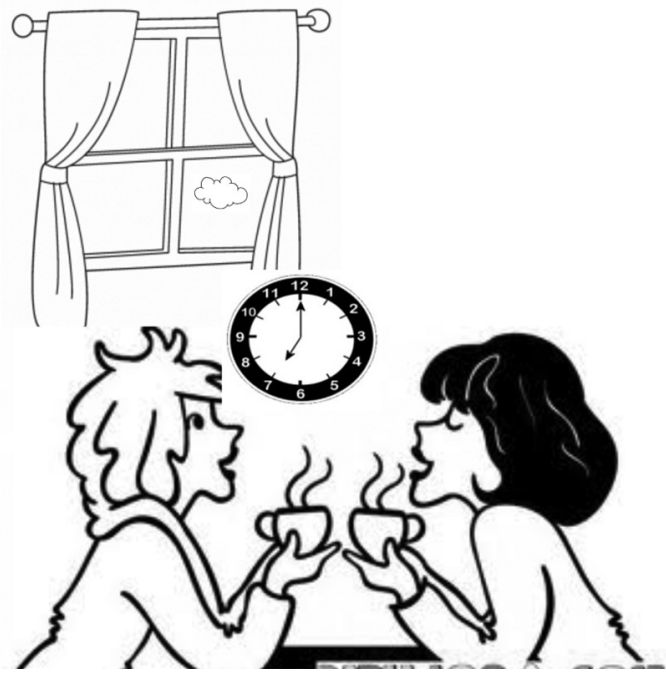

Figura 2. Tomado de http://www.dibujos.com

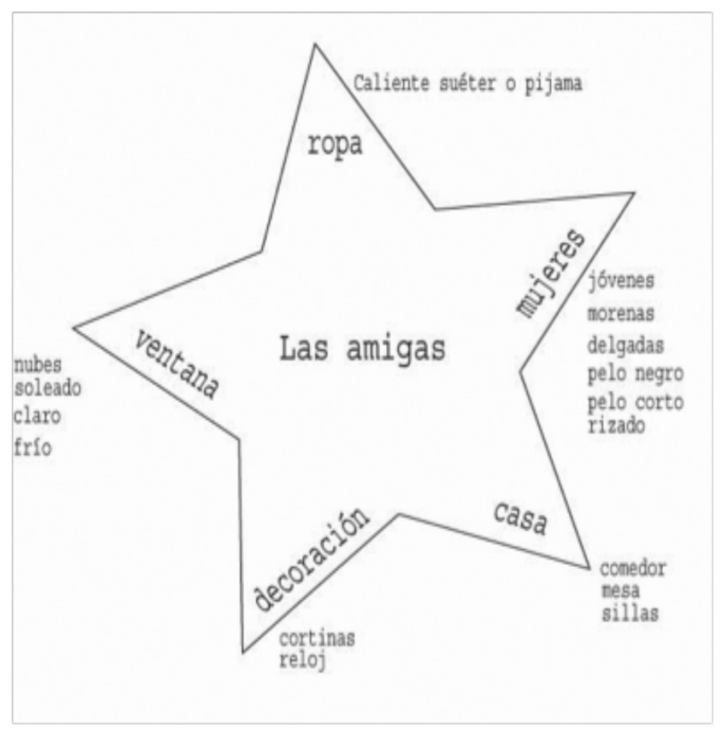

Figura 3. Ejemplo de estrella de palabras. Autora, con base en el trabajo de un estudiante 
Una vez analizada la tarjeta, el estudiante procedió a generar ideas mediante una estrella de cinco picos tal como se muestra en el ejemplo. Luego elaboró el siguiente párrafo:

En la sala de la casa están dos amigas hablando. Quizás sean hermanas gemelas, porque físicamente son muy parecidas: mujeres jóvenes, con pelo corto y rizado, ojos redondos y cuello largo y delgado. Ellas están hablando sobre las noticias que aparecen en el periódico de ese día. Según el reloj que está en la pared son las siete de la mañana. En la sala hay una ventana que tiene las cortinas abiertas. A través de la ventana se puede ver una nube blanca, pero la mayor parte del cielo está despejado. Quizás hace frío, porque las mujeres tienen ropa caliente: abrigos o pijama; además una de las chicas está sosteniendo una taza de alguna bebida, puede ser café o chocolate.

Una variación de esta figura es la estrella de seis picos. Según Cassany, el gráfico de la estrella se deriva de la fórmula periodística de la noticia, según la cual, para informar de un hecho, tiene que especificarse el quién, el qué, el cuándo, el dónde, el cómo y el porqué. "Estos seis puntos, las llamadas 6Q, son esenciales de cualquier tema, aunque pueden ampliarse con otras interrogaciones" (Cassany 1993: 64).

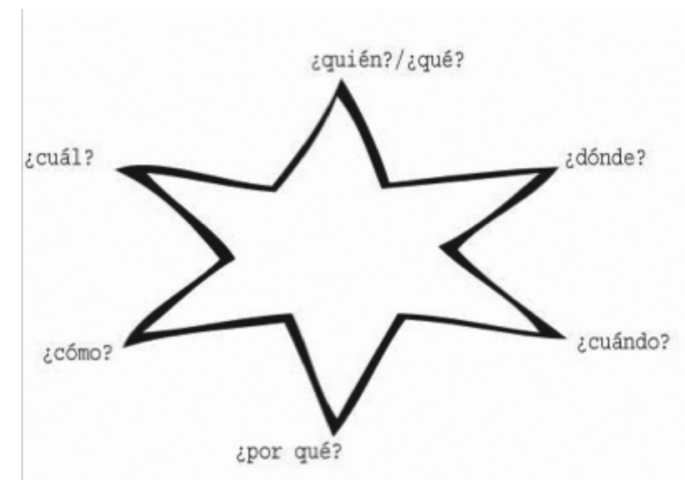

Figura 4. Ejemplo de estrella de seis picos Autora, con base en el trabajo de un estudiante

Para desarrollar el ejercicio con este modelo de estrella, se utilizó una fotografía de Jennifer López, la cual contenía la oración: Jennifer López es la mejor actriz latina de la historia por su belleza y su talento, también es una de las mejores cantantes del mundo latino.

A partir de este material, los estudiantes empezaron a generar ideas contestando las preguntas escritas en cada punta de la estrella:

¿Quién es?: Jennifer López es una cantante y actriz muy famosa nacida en New York, pero de familia puertorriqueña.

¿Dónde es famosa?: es famosa tanto en Estados Unidos como en Latinoamérica.

¿Cuándo ocurrió eso?: después de filmar una película cuando interpretó a la cantante Selena. ¿Por qué se hizo famosa?: tiene mucho talento para el baile, el canto y la actuación.

¿Cómo lo hizo?: estudiando baile y practicando mucho.

¿Cuáles películas filmó y cuáles canciones cantó?: ha filmado varias películas y ha grabado varios álbumes musicales de temas en inglés y en español. 
El párrafo redactado resultó de la siguiente manera:

Jennifer López es una actriz y cantante muy famosa tanto en Estados Unidos como en Latinoamérica. Ella estudió canto y baile y se hizo famosa después de una audición donde tenía que interpretar el papel de la cantante Selena. Ha filmado varias películas importantes y ha grabado algunos álbumes de música en inglés y en español. Por todo eso, Jennifer López es la mejor actriz y cantante latina de la historia tanto por su belleza como por su talento.

\subsubsection{La rueda de palabras}

Como en el diagrama de la estrella, la rueda de palabras es útil para reunir tanto vocabulario como se piensa sobre el tema. Este gráfico puede ser un círculo o un óvalo. En el centro del círculo u óvalo, se escribe una palabra o una frase corta que es importante para la redacción del tema. Luego se dibujan líneas y, al final de cada una de ellas, se escribe un vocablo que se asocia con el del centro (Gerrard y Spaine 1993). Una variación es el llamado Modelo del escarabajo llamado así por dos razones:

La primera, porque el esquema simplificado de su figura nos permite trabajar con una herramienta que facilita notablemente el proceso de escritura. La segunda, tiene un sentido figurado o de naturaleza simbólica: así como el escarabajo escarba y hurga en la tierra, debemos hacerlo en nuestra mente para encontrar las redes asociativas de la palabra o del tema. (Chávez 1998: 193)

El ejemplo muestra cómo se realizó esta técnica, cuyo propósito era la redacción de un párrafo a partir de la frase "El invierno en Costa Rica". Como se aprecia en el diagrama, el estudiante escribió el tema en el centro y comenzó a hacer relacionar ideas. Luego, escribió el párrafo.

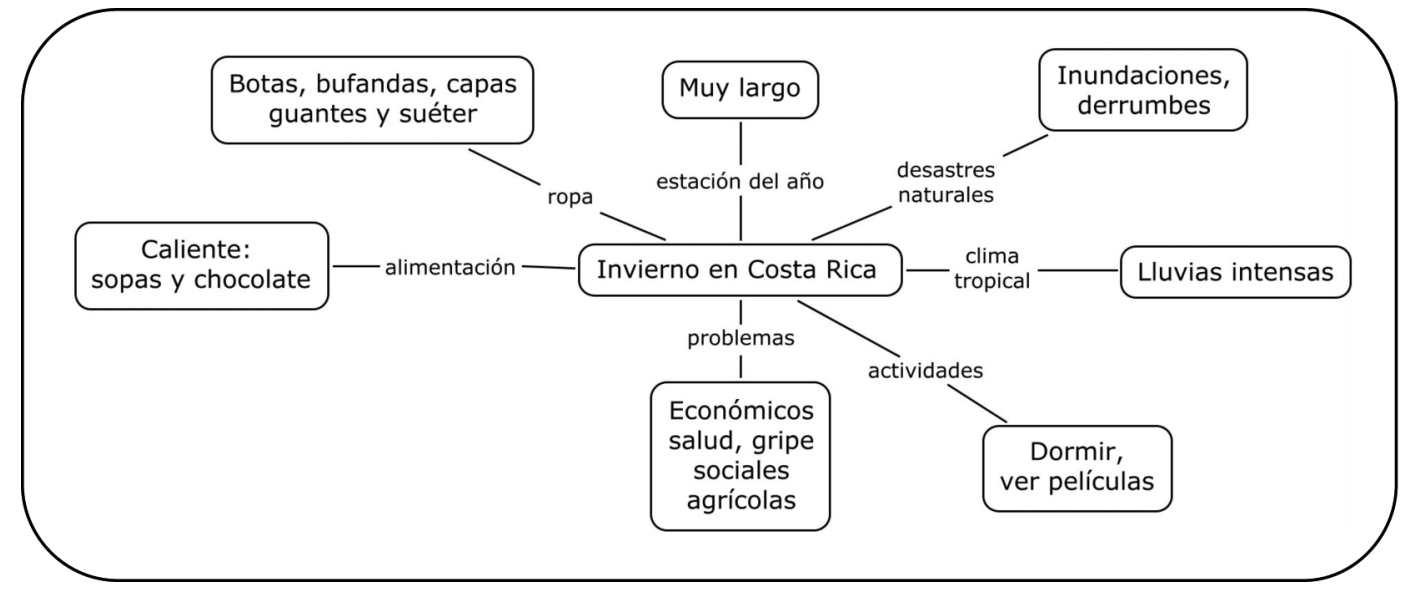

Figura 5. Ejemplo modelo del escarabajo

Autora, con base en el trabajo de un estudiante

El invierno en Costa Rica es característico de la zona tropical, con presencia de lluvias intensas en un periodo que dura cerca de ocho meses. Como las lluvias son muy intensas provoca diferentes y graves problemas sociales y económicos porque hay inundaciones que hacen que se pierda la producción agrícola como la cosecha del café y legumbres y también hay derrumbes interrumpen el libre tránsito por las carreteras del país. Además hay problemas en la salud, ya que 
es muy común que las personas se enfermen de gripe, por eso es importante vestirse con ropa adecuada como botas, suéter, bufandas y capas. Por otra parte, el invierno hace que la gente cambie sus hábitos de vida, por ejemplo, hacer más actividades adentro de las casas, ver televisión, comer comidas calientes como sopas y chocolate y dormir. Eso es lo mejor de esta época del año.

\subsubsection{El árbol de ideas}

Una vez que se tengan algunas ideas con las cuales se quiera trabajar, se puede generar más por medio del árbol de ideas (Gerrard y Spaine: 1993). Un gráfico de esta forma es un esquema útil para mostrar la relación entre las ideas: la general se escribe en la parte superior y las secundarias se van extendiendo hacia abajo. De esta manera, se obtiene un mapa visual de todas las relaciones que se han generado.

Esta técnica ofrece dos ventajas: por un lado, el autor puede darse cuenta, casi de inmediato, de si ha omitido alguna idea relevante en el proceso de la textualización; por otro lado, cuando se examine la información obtenida, pueden surgir otras ideas que podrían incluirse, es decir, que el autor puede visualizar nuevas ramas para agregar al árbol.

Un ejemplo creado por un estudiante en la clase dio como resultado el siguiente árbol de ideas; el ejercicio consistía en redactar un párrafo descriptivo con el tema ¿Quién soy yo?

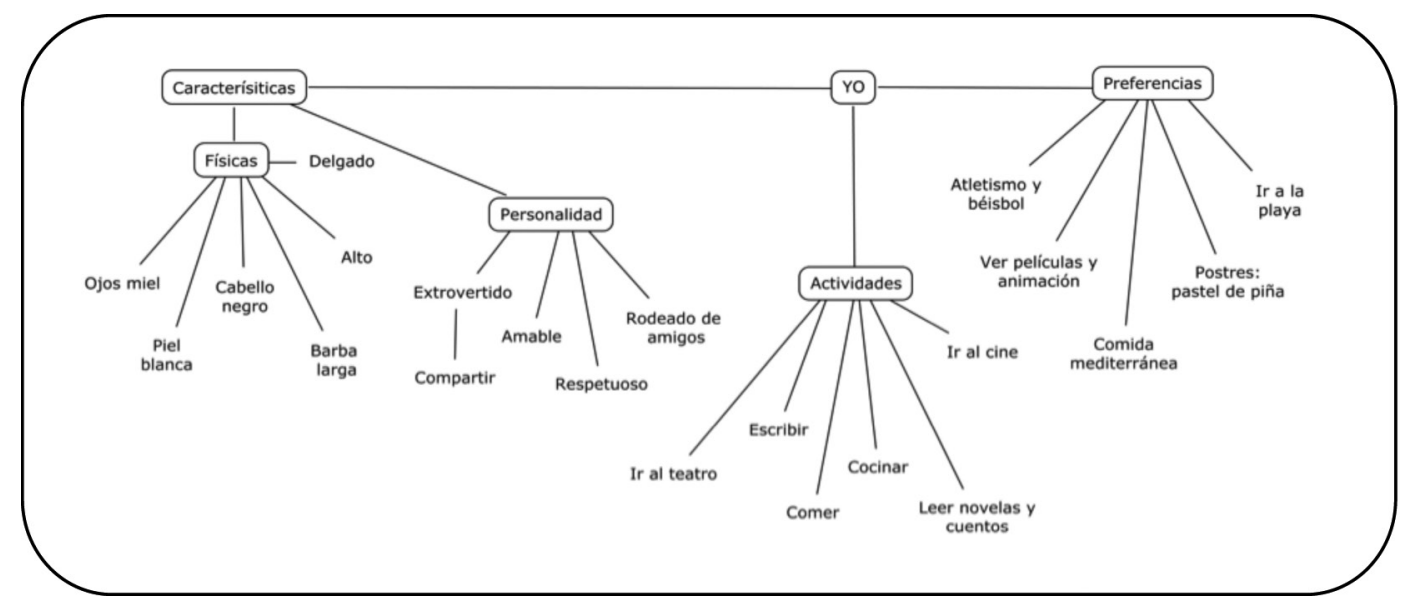

Figura 6. Ejemplo del árbol de ideas

Autora, con base en el trabajo de un estudiante

Después de elaborar el esquema, el estudiante desarrolló el párrafo de esta manera:

Me llamo Sebastián. Soy un muchacho delgado, alto, de piel blanca y cabello café. Tengo ojos color miel y uso la barba larga. Soy muy extrovertido por eso siempre estoy rodeado de amigos. Trato de ser amable hasta con quienes no conozco pues considero que la empatía y el respeto son buenas cartas de presentación. Soy muy activo y entre las actividades que más me gusta hacer está cocinar, especialmente comida mediterránea y postres. Mi favorito es el pastel de piña. Otras actividades que realizo son correr e ir al cine y al teatro; las películas animadas son mis preferidas. Me gusta también tener siempre a mi lado una buena novela para leer y un papel, ya que escribo mis propias historias desde que tenía nueve años. 
Me encanta pasear y mis paseos favoritos son los que hago a la playa. Practico atletismo y béisbol pero ahora no los practico tanto como cuando estaba en el colegio. Más o menos, ese soy yo.

\subsubsection{El cubo}

Esta forma de explorar ideas consiste en estudiar las seis caras posibles de un hecho a partir de seis puntos de vista (Cassany 1993). El procedimiento consiste en dibujar un cubo de seis lados, a cada uno de ellos le corresponde una pregunta, cuyas respuestas servirán de base para el desarrollo del tema. Las preguntas son: Describir: ¿cómo se ve?, ¿cómo se siente, se huele o se toca?, ¿a qué sabe? Comparar: ¿a qué se parece?, ¿en qué se diferencia? Relacionar: ¿con qué se relaciona? Analizar: ¿cuántas partes tiene?, ¿cuáles son esas partes?, ¿cómo funcionan?, ¿qué ventajas y desventajas tiene? Aplicar: ¿para qué sirve?, ¿cómo se usa? Argumentar: ¿cuáles ventajas o desventajas tiene?

El siguiente ejemplo muestra la técnica desarrollada a partir de una imagen, en este caso, un avión.

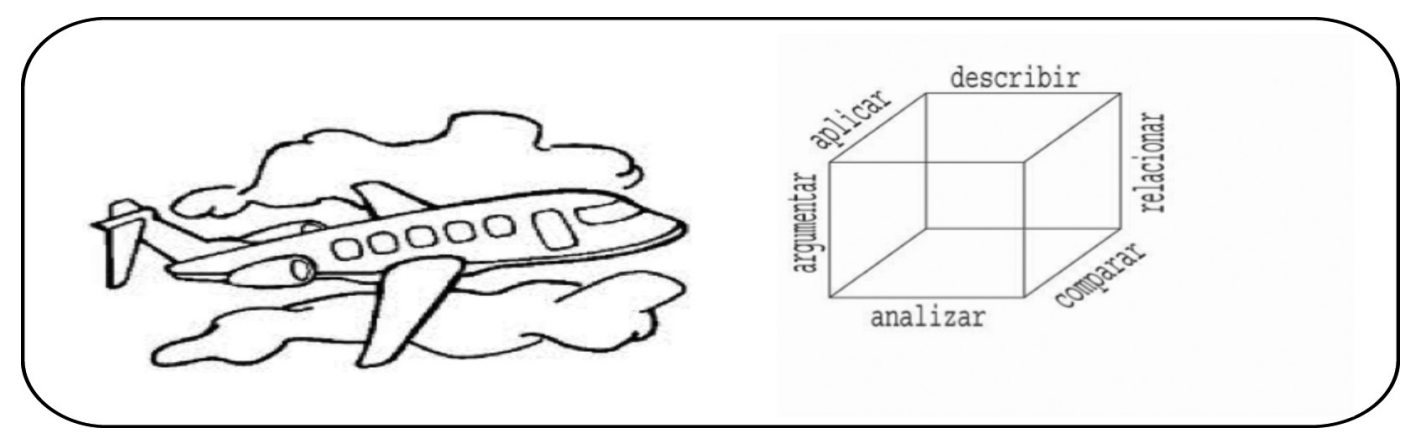

Figura 7. Ejemplo del cubo.

Tomado de http://quedibujos.com/aviones

El resultado del ejercicio fue el siguiente:

Describir: avión grande de pasajeros, bonito, grandes nubes, se ven las ventanas y una puerta, es plateado con negro. Se ven dos alas y la cola.

Analizar: avión, dos turbinas, dos alas, ventanas y una puerta, la cola y la ventana del piloto, dos nubes grandes.

Comparar: avión, bus, tren, carro.

Relacionar: paseos, turismo, otros países, vacaciones.

Aplicar: viajar, medio de transporte.

Argumentar: rápido, seguro, emocionante, caro.

El párrafo elaborado resultó así:

La tarjeta presenta un avión de pasajeros, es grande y bonito. Su color es plateado con rayas negras. Se ven las ventanas a través de las cuales los pasajeros pueden ver las grandes nubes que rodean el avión. También se pueden apreciar las turbinas o los motores y la nave tiene la nariz hacia arriba lo que da la idea de que está en 
pleno vuelo. Este aparato se usa para volar a grandes alturas y de esta manera poder ir a lugares lejos, es decir, cuando se mira la imagen de inmediato se hace una relación con otros países, con viajes, con vacaciones en lugares excitantes donde haya magníficas playas las de Brasil. Este medio de transporte es más caro que el bus o el tren, pero es más seguro, más rápido y más emocionante. Nunca me he montado en un avión, pero no pierdo la esperanza de hacerlo algún día, ojalá en un tiempo cercano.

\subsubsection{Develar palabras clave}

Muchas veces, cuando se plantea una idea se cree que esta contiene toda la información necesaria para ser comprendida. Sin embargo, detrás de cada vocablo es posible que se escondan otros que poseen una importante carga informativa fundamental para la comprensión del texto (Cassany 1993).

Una forma de extraer más información es develando las palabras clave. Primero, se identifican esas palabras; luego, por medio de preguntas, se realiza un listado de posibles respuestas. Finalmente, se reescribe el texto con la nueva información. Con esta técnica, se pueden explorar más ideas con la finalidad es expandir la escritura. Es muy útil en la elaboración de oraciones temáticas y formulación de tesis. Con esta técnica, el estudiante realizó el siguiente ejercicio:

La muchacha trabajó en una compañía de seguros por mucho tiempo; era buena trabajadora, pero tuvo problemas con su jefe.

- Muchacha joven: joven, bonita, inteligente, entusiasta.

- Trabajó: duro, cinco años, 10 horas diarias, horas extra.

- Compañía: institución, empresa, pública.

- Seguros: automóviles, casa, incendios, salud, desastres.

- Buena: responsable, puntual, ordenada, respetada.

- Problema: acoso sexual, laboral, salud, rendimiento, renuncia.

- Jefe: vieio. acosador. irrespetuoso.

Figura 7. Ejemplo de palabras clave.

Autora, con base en el trabajo de un estudiante

Al final, el párrafo escrito resultó de este modo:

La muchacha era joven, bonita y muy inteligente. Trabajó en una compañía de seguros de automóviles privada durante cinco años, pero un día dejó de hacerlo porque tuvo un problema con su jefe. Este era un viejo acosador e irrespetuoso, pero nunca había hecho nada hasta ese día en que trató de besarla. Ella renunció de inmediato. Fue una gran pérdida para la empresa pues era muy buena trabajadora y tenía muchas cualidades, entre las que se pueden mencionar: puntual, responsable y muy ordenada. Además, siempre trabajaba con mucho entusiasmo aunque su horario era de más de diez horas diarias y también horas extras. 


\section{Conclusiones}

En síntesis, el proceso de escritura es una actividad compleja y requiere de mucha práctica. No es una habilidad espontánea como conversar, pues el escritor construye el texto con trabajo y oficio: reflexiona sobre la situación comunicativa, apunta ideas, hace esquemas, redacta borradores, repasa pruebas (Cassany 2000). En otras palabras, cada circunstancia nueva de producción o de interpretación requiere un esfuerzo de adecuación que necesita lectura, reflexión, pruebas y revisiones. Esta complejidad, en ocasiones, provoca tanta tensión que los escritores no encuentran con facilidad la manera de empezar a escribir el texto. Puede ser que tengan mucha información y no sepan cómo organizarla o que, por el contrario, las ideas falten y no puedan encontrar un recurso que les ayude a generarlas.

Por lo general, los estudiantes que asisten a los cursos de redacción creen que escribir es poner en un papel las ideas y los pensamientos sobre un tema tal como van llegando a la mente con el único propósito de cumplir con la tarea de redactar el texto requerido. No se detienen a reflexionar sobre su estructura o sobre su contenido; en consecuencia, el escrito resulta desorganizado, sin cohesión ni coherencia.

Otras veces, les resulta muy difícil encontrar las ideas o no son lo suficientemente hábiles para desarrollarlas. Por ello, es necesario enseñarles diversas estrategias que los motiven a ser más creativos, a indagar más sobre su escrito, a escribir más información que solo listas de palabras u oraciones.

Los recursos expuestos y ejemplificados en este trabajo demuestran que, con el empleo de estas técnicas en la etapa de la preescritura, los estudiantes adquieren la destreza de la escritura como un proceso en el cual la planificación es fundamental no solo para lograr un escrito organizado y con sentido, sino cumple con el propósito para el que fue creado.

\section{Bibliografía}

Arley Fonseca, Mauricio. 2010. El arte de escribir. Heredia: EUNA.

Bolaños, Bolívar. 2002. Comunicación Escrita. San José: EUNED.

Brooke, Arthur y P. Grundy. 1998. Beginnig to write: writing activities for elementary and intermediate learners. Cambridge: Cambridge University Press.

Calsamiglia, Helena y Amparo Tusón. 1999. Las cosas del decir. Manual de análisis del discurso. Barcelona: Editorial Ariel, S.A.

Cassany, Daniel. 1989. Describir el escribir. España: Ediciones Paidós Ibérica, S.A.

1993. La cocina de la escritura. Barcelona: Editorial Anagrama, S.A.

2000. Reparar la escritura. Didáctica de la corrección de lo escrito. Barcelona: Editorial Grao.

Chávez Pérez, Fidel. 1998. Redacción avanzada. Un enfoque lingüístico. México: PEARSON Educación. 
Figueras, Carolina y Marisa Santiago. 2000. “Planificación”. En: Montolío (coord.), 15-67.

Flower, Linda y John Hayes (1981). "A Cognitive Process Theory of Writing”. College Composition and Communication, 32 (4): 365 - 387.

Gerrard, Lisa y Sheri Spaine Long. 1993. Redacción y revisión: Estrategias para la composición en español. Unites States of America: McGraw-Hill, Inc.

Madrigal Abarca, Marta. 2005. La enseñanza de la escritura como proceso a estudiantes de español como segunda lengua de nivel avanzado. Tesis de maestría en español como segunda lengua: Universidad de Costa Rica.

2008. "La escritura como proceso: metodología para la enseñanza de la expresión escrita en español como segunda lengua”. Revista de Filología y Lingüística, 34 (1): 129-141.

Merino, María Eugenia. 2007. Escribir bien, corregir mejor: corrección de estilo y propiedad idiomática. México: Editorial Trillas.

Montolío, Estrella. 2000. Manual práctico de escritura académica. Vol. II. Barcelona: Editorial Ariel, S.A.

Que dibujos!! 2011. http://www.quedibujos.com

Valdés, Guadalupe, Trisha Dvorak y Thomasina Pagán Hannum. 1998. Composición, proceso y síntesis. USA: McGraw-Hill Companies, Inc. 
